\title{
Absceso de ovario causado por Streptococcus bovis en una paciente con cáncer de colon no diagnosticado
}

\author{
CARMEN NOBILE B. ${ }^{1}$, MYRIAN FIGUEROA L. ${ }^{1}$
}

\section{Ovarian abscess due to Streptococcus bovis in a patient with unnoticed colonic cancer}

Streptococcus bovis is a member of the human gut flora. The most important clinical infections caused by $S$. bovis are bacteremia and endocarditis; usually bacteremia has been described in association with bowel pathology. Infections, although quite rare, can in some instances be the sole clue to the presence of malignancy. These infections are related to either invasion of tissues or organs that are in close proximity to the tumor, or may be secondary to distant seeding, arising through transient bacteremia caused by necrotic tumor tissue. In this report, we describe a case of a patient with an ovarian abscess caused by S. bovis. During the treatment the patient was found to have a colonic malignant lesion.

Key words: Colonic neoplasm; Abscess; Streptococcus bovis.

\section{Introducción}

Streptococcus bovis es un estreptococo del Grupo D. Las infecciones más frecuentes causadas por este microorganismo que forma parte de la flora intestinal humana son bacteriemias y endocarditis ${ }^{1,3,5}$, la primera asociada con patología intestinal ${ }^{11}$.

El hallazgo de $S$. bovis en distintas infecciones clínicas es relativamente benigno, pero acentúa la frecuencia y severidad potencial de las lesiones asociadas al colon, requiriendo colonoscopia e instauración de tratamiento antimicrobiano específico y de la lesión colónica de alto riesgo en forma mandatario.

Como se ha reportado en publicaciones pre$v \cos ^{2}$, la existencia de lesiones polipoideas en colon hace probable que ésta sea la puerta de entrada del Streptococcus bovis.

\section{Caso clínico}

Paciente con 48 años de edad, sexo femenino, con antecedentes de diabetes tipo II de 10 años de evolución. Ingresó con pérdida de peso, vó- mitos, cambios en el hábito intestinal (sin sangre, ni melena), dolor en flanco derecho, e ictericia.En los análisis bioquímicos destacó la presencia de anemia y leucocitosis con desviación izquierda. El diagnóstico inicial fue de infección de la cavidad abdominal.

Se inició terapia antimicrobiana empírica con ampicilina, gentamicina y metronidazol. En una ecografía ginecológica se observó una imagen oval de bordes nítidos de 71 x 55 mm en la región parauterina derecha y en el fondo uterino se apreciaba otra imagen de contenido líquido (de 84 x $40 \mathrm{~mm}$ ), ambas de naturaleza incierta. En el tacto rectal se palpó una tumoración de consistencia dura a $7 \mathrm{~cm}$ del esfínter anal. La TAC abdominal demostró engrosamiento de la pared de ciego y colon ascendente con estenosis a dicho nivel, adenopatías retroperitoneales periaórticas y una masa multilobulada con contenido heterogéneo y gruesos tabiques. Se hizo el diagnóstico imagenológico presuntivo de absceso intrapelviano sin poder descartar citoadenoma de ovario. La paciente fue sometida a una laparotomía exploratoria en la que se comprobó la existencia un tumor ovárico de $8 \mathrm{~cm}$ en el saco de Douglas. Se obtuvieron muestras del líquido de punción y

1 Hospital Nuestra Sra. de la Misericordia. Córdoba. Argentina.

Recibido: 22 enero 2003

Aceptado: 29 abril 2003 
Tabla 1. Características de la cepa de Streptococcus bovis I

\begin{tabular}{lc}
\hline Características & Resultado \\
\hline Vancomicina & $\mathrm{S}$ \\
Catalasa & - \\
Hemólisis en agar sangre de cordero 5\% & - \\
Crecimiento en $\mathrm{NaCl}$ 6,5\% & - \\
PYR & - \\
Movilidad & - \\
Bilis esculina & + \\
Arginina & - \\
Voges Proskauer & + \\
Urea & - \\
Manitol & + \\
Sorbitol & - \\
Amilasa & + \\
Arabinosa & + \\
Rafinosa & - \\
\hline
\end{tabular}

tejido siendo remitidas a estudio histopatológico y microbiológico. La anatomía patológica informó: "la formación quística está revestida por exudado leucocitario con plasmocitos y la pared es fibrosa. No se observan proliferaciones neoplásicas malignas." Los hallazgos bacteriológicos fueron los siguientes: en la tinción de Gram directa se observó abundantes polimorfonucleares, cocos Gram (+)s en diplo y en cadenas, y bacilos Gram (-)s. En el cultivo de 24 horas se desarrollaron cocos Gram (+)s tipificados como Streptococcus bovis I (Tabla 1) y bacilos Gram (-)s identificados como Enterobacter cloacae. Estudios de susceptibilidad según Kirby y Bauer y criterios de NCCLS. Streptococcus bovis: susceptible a cloranfenicol, cotrimoxazol, tetraciclina, gentamicina, rifampicina, eritromicina. No se evaluaron penicilina y ceftriaxona por no disponerse de medios para realizar CIM. Con el informe de laboratorio se adecuó el esquema antimicrobiano introduciendo imipenen y suspendiendo metronidazol, con lo cual se observó una respuesta clínica y resolución favorables.

El hallazgo de $S$. bovis I y su relación con patologías malignas, así como el resultado del antígeno carcinoembrionario de 40,3 U/ml (VN: $<35 \mathrm{U} / \mathrm{ml}$ ), alertó al equipo de salud sobre la posibilidad de otra patología subyacente no diagnosticada. Se le realizó una colonoscopia y biopsia que reveló una imagen sospechosa de tumor y posteriormente el estudio histopatológico e inmunohistoquímico de la muestra confirmó la existencia de un adenocarcinoma de colon ${ }^{13,17}$.

\section{Discusión}

Streptococcus bovis ha sido identificado como agente causal de severas infecciones humanas, incluyendo endocarditis y bacteriemias ${ }^{1,3,5,6,9}$, aunque excepcionalmente se han descrito casos de meningitis y sepsis neonatales ${ }^{2,4,8}$. Habitualmente las infecciones se presentan en individuos inmunosuprimidos y/o con lesiones en el tracto digestivo: pólipos o neoplasias ${ }^{2,6,7,10}$. La tasa de portación fecal de $S$. bovis está incrementada en pacientes con lesiones colónicas premalignas y malignas, comparadas con controles sanos y pacientes con enfermedades gastrointestinales no malignas ${ }^{11,13}$.

Tradicionalmente, las infecciones bacterianas no han sido consideradas como la principal causa de cáncer. Sin embargo, las bacterias están relacionadas al cáncer por dos mecanismos: inflamación crónica y producción de metabolitos carcinogénicos. La producción de citoquinas inflamatorias tales como: FNT- $\alpha$, IL - $1 \beta$, IL-6 e IL-8, producen la formación de óxido nítrico y radicales libres como superóxido, peroxinitritos, radicales hidroxilos y radicales alkilperóxidos, contribuyendo a los procesos neoplásicos por alteración del ADN celular. Por otro lado, la producción en la mucosa colónica de IL-8, similar a factores angiogénicos gatillados por antígenos de la pared de $S$. bovis, puede también favorecer la progresión de carcinogénesis de colon ${ }^{12}$.

Se han reportado muchas infecciones asociadas a cáncer colorectal ${ }^{1,5,7}$, aquí se describe un caso de una paciente con un absceso ovárico causado por $S$. bovis y con cáncer de colon no diagnosticado.

En este caso la infección estaría relacionada a la invasión de tejidos u órganos que se encuentran próximos al tumor, o podría ser secundaria a una siembra a distancia a través de la bacteremia transitoria originada en el tejido tumoral necrótico ${ }^{7}$.

Se ha sugerido que la acción local de citoquinas o de mediadores químicos capaces de promover vasodilatación y aumento de la permeabilidad capilar en el sitio del neoplasma, permitiría la proliferación de $S$. bovis y su paso a la circulación sanguínea. Otros posibles puntos de origen del microorganismo serían focos sépticos genitourinarios o dentales ${ }^{12}$.

Es destacable la importancia de la recuperación de esta especie bacteriana en cultivos de sangre o de tejidos, ya que puede indicar la presencia de un neoplasma no diagnosticado, oculto, subyacente.

Desde el punto de vista microbiológico resulta fundamental realizar una segura identificación del 
microorganismo diferenciándolo de Streptococcus grupo viridans y de Enterococcus sp para elegir la más apropiada terapia antimicrobiana. Se recomienda también la diferenciación entre los dos biotipos de $S$. bovis, por la habilidad del primero de producir dextranos, fermentar manitol y degradar almidón. El biotipo I es más virulento que el biotipo II y se asocia en mayor porcentaje a pacientes con lesiones colónicas ${ }^{10}$.

\section{Resumen}

Streptococcus bovis forma parte de la flora intestinal humana. Las infecciones clínicas más importantes causadas por este microorganismo son bacteriemia y endocarditis, estando la primera asociada con patologías intestinales, especialmente con enfermedades malignas como cáncer o poliposis de colon. Las infecciones de diversos sitios anatómicos, aunque infrecuentes, pueden en ciertas instancias ser la sola clave de la presencia de malignidad. Estas infecciones se relacionan con la invasión de tejidos u órganos próximos al tumor o pueden ser secundarias a una siembra a distancia, a través de la bacteriemia originada en tejido tumoral necrótico. En este reporte se describe el caso de una paciente con un quiste de ovario abscedado en el que se aisló Streptococcus bovis I y Enterobacter cloacae. Durante el tratamiento se diagnosticó cáncer de colon.

\section{Bibliografía}

1.- Anaf V, Noel J C, Thys J P, Simon P, Buxant F. A first case of Streptococcus bovis bacteremia and peritonitis from endometrial cancer origin. Acta Chir Belg 2001; 101 (1): 38-9
2.- Barragan-Casas J, Arroyo-Burgillo P, Sanz-Rojas P, Serrano-Heranz R, Sánchez-Fuentes D. Meningitis por Streptococcus bovis biotipo II. Caso clínico y revisión de la literatura. Enferm Infecc Microbiol Clin 2002; 20: $537-8$

3.- González-Quintela A, Martínez-Rey C, Castroagudin J F, Rajo-Iglesias M C, Dominguez-Santalla M J. Prevalence of liver disease in patients with Streptococcus bovis bacteremia. J Infect 2001; 42 (2): 116-9.

4.- Vilarrasa N, Prats A, Pujol M, Gasón A, Viladrich F. Streptococcus bovis meningitis in a healthy adult patient. Scand J Infect Dis 2001; 34 (1): 61-2.

5.- Sajkowska A, Wykretowicz A, Minczykowki A, Smielecki J, Wysocki H. Infective endocarditis due to Streptococcus bovis in a patient with asymptomatic colon cancer -a case report. Polish Heart J 2002; 56 (5): 538

6.- Reilly J, West J, Still C. Splenic abscess and a large villous adenoma associated with Streptococcus bovis endocarditis. Contemporary Surg 2001; 57 (6): 300-4.

7.- Osada $T$, Nagawa H, Masaki $T$ et al. Thoracic empyema associated with recurrent colon cancer. Dis Colon Rectum 2001; 44: 291-4.

8.- Grant R, Whitehead T, Orr J. Streptococcus bovis meningitis in an infant. J Clin Microbiol 2000 Jan; 38 (1): 462-3.

9.- Duval X, Papastamopoulos V, Longuet P et al. Definite Streptococcus bovis endocarditis: characteristics in 20 patients. Clin Microbiol Infect 2001; 7: 3-10.

10.- Thota P, Sanaka M, Conwell D. A 76-year-old man with septic arthritis. Cleveland Clin J Med 2002; 69: 549-53.

11.- Klein R S, Recco R A, Catalano M T, Edberg S C, Casey J I, Steigbigel N H. Association of Streptococcus bovis with carcinoma of the colon. N Engl J Med 1977; 297: 800-2.

12.- Ellmerich S, Schöder M, Duranton B et al. Promotion of intestinal carcinogenesis by Streptococcus bovis. Carcinogenesis 2000; 21 (4): 753-6.

13.- Groves C. Johns Hopkins Microbiology Newsletter 1997; 16 (42).

14.- The American Society of colon and rectal surgeon's practice parameter for detection of colorectal neoplasms . Dis Colon Rectum 1999; 42: 1128-9.

15.- Stzkorn K. Colon cancer in the 2000's BorlandGroover Clinic Colon Cancer in the 2000's.

Correspondencia a:

Myrian Figueroa L.

E-mail: myrianfigueroa@hotmail.com 